\title{
Microthermometric characteristics of vein type U-Th enrichment observed in the Fault Zones in Northwest of Arıklı (Çanakkale-Turkey)
}

\author{
Arıklı (Çanakkale-Türkiye) kuzeybatısında fay zonlarında gözlenen damar tipi U-Th \\ zenginleşmesinin mikrotermometrik özellikleri
}

\author{
Sercan ÖZTÜRK ${ }^{1, a}$, Cihan YALÇIN ${ }^{* 2, b}$, Mustafa KUMRAL ${ }^{1, c}$ \\ ${ }^{1}$ Istanbul Teknik Üniversitesi, Maden Fakültesi, Jeoloji Mühendisliği Bölümü, 34100, İstanbul \\ ${ }^{2}$ Sanayi ve Teknoloji Bakanlığı, Sanayi Bölgeleri Genel Müdürlüğü, 06100, Ankara
}

\begin{abstract}
• Geliş tarihi / Received: 21.01.2021 • Düzeltilerek geliş tarihi / Received in revised form: $12.04 .2021 \quad$ • Kabul tarihi / Accepted: 21.04 .2021
\end{abstract}
\begin{abstract}
U-Th enrichments are observed in many regions in Western Anatolia. There are also U and Th enrichments in the fault zones developed in the tuffs in Arıklı in Biga Peninsula. The characterization of the mineralization is vein type and epigenetic formation. Çetmi Ophiolitic Melange forms the basis of the region. Küçükkuyu formation, which consists of Arıklı tuff member and shale-sandstone member, is located on this unit. The youngest unit in the region is diabase dykes. The study area was affected by deformations during the Paleogene period and E-W and NE-SW extended dip slip fault systems were developed. In the fault zone around Örencik Tepe, 64-1640 ppm values for U and 302-11813 ppm values for Th were obtained respectively. At the same time, hydrothermal magnesite breccias developed in this fault zone. Because of microthermometric assessments in these breccias associated with mineralization, it was determined that the homogenization temperatures $\left(\mathrm{Th},{ }^{\circ} \mathrm{C}\right.$ ) were between $282-348{ }^{\circ} \mathrm{C}$ and the $\% \mathrm{NaCl}$ salinity equivalents were between 4.2 8.0 respectively. It was identified that the solution system of the liquid inclusions is as $\mathrm{H}_{2} \mathrm{O}-\mathrm{MgCl}_{2}-\mathrm{CaCl}_{2}$ and the density of the liquids is between $0.58-0.74 \mathrm{~g} / \mathrm{cm}^{3}$.
\end{abstract}

Keywords: Arıklı tuff member, Hydrothermal magnesite, Microthermometric measurements, Vein type U-Th mineralization

$\ddot{O} z$

Batı Anadolu'da birçok bölgede U-Th zenginleşmeleri gözlenir. Biga Yarımadası sinırları içindeki Arıklı ve civarındaki tüflerde gelişen fay zonlarinda da $U$ ve Th zenginleşmeleri bulunur. Cevherleşme damar tipi şeklinde ve epijenetik oluşumludur. Bölgenin temelini Çetmi Ofiyolitik Melanjı oluşturur. Bu birim üzerinde Arıkll tüf üyesi ve şeyl-kumtaşı üyesinden oluşan Küçükkuyu formasyonu yer alır. Bölgedeki en genç birim ise diyabaz dayklarıdır. İnceleme alanı Paleojen dönemindeki deformasyonlardan etkilenmiş ve $E$-W ve NE-SW uzanıml eğim atıml fay sistemleri gelişmişstir. Örencik tepesi civarındaki fay zonunda U için 64-1640 ppm ve Th için ise 302-11813 ppm değerleri elde edilmiştir. Aynı zamanda bu fay zonunda hidrotermal magnezit breşleri gelişmiştir. Cevherleşme ile ilişkili olan bu breşlerde mikrotermometrik ölçümler sonucunda homojenleşme sicakliklarının $\left(\mathrm{Th},{ }^{\circ} \mathrm{C}\right) 282-348^{\circ} \mathrm{C}$ arasında olduğu ve \% $\mathrm{NaCl}$ tuzluluk eşdeğerlerinin de 4.2-8.0 arasında olduğu belirlenmiştir. Sivı kapanımların çözelti sisteminin $\mathrm{H}_{2} \mathrm{O}-\mathrm{MgCl}_{2}-\mathrm{CaCl}_{2}$ şeklinde olduğu ve siviların yoğnuluğunun da $0.58-0.74 \mathrm{~g} / \mathrm{cm}^{3}$ arasında olduğu ortaya çıkarllmıştır.

Anahtar kelimeler: Arıklı tüf üyesi, Hidrotermal manyezit, Mikrotermometrik ölçümler, Damar tipi U-Th cevherleşmesi

\footnotetext{
*b Cihan YALÇIN; cihanyalcinjeo@gmail.com, Tel: (0535) 77305 73, orcid.org/ 0000-0002-0510-2992

${ }^{\mathrm{a}}$ orcid.org/ 0000-0003-4478-2908 $\quad{ }^{\mathrm{c}}$ orcid.org/ 0000-0001-7827-8721
} 


\section{Introduction}

Uranium and Thorium are important radioactive elements. Turkey has significant uranium enrichment in western Anatolia (Şaşmaz, 2008). The International Atomic Energy Agency (IAEA, has classified the uranium deposits into 14 groups (Anonymous, 2009). This classification was made according to age, tectonic, origin rock and location. Granitic and volcanic rocks constitute important lithologies for uranium enrichment (Zhang and Zhang, 1991; Qin and Liu, 1998). According to the wall rocks, $\mathrm{U}$ deposits are divided into 4 groups as granite-rock type (G-type), volcanic-rock type (Vtype), carbon-silica-pelitic-rock type (CSP-type) and sandstone type (Li et al., 2002).

Uranium exploration in Turkey was initiated by General Directorate of Mineral Research and Exploration (MTA) in 1953 and has continued to work after (the Contencin, 1960; Uçmak, 1969; Kaplan, 2011; Yörükoğlu, 2014, Günaydın, 2017). $\mathrm{U}_{3} \mathrm{O}_{8}$ value is $0.08 \%$ in Miocene aged carbonated rocks in Ayvacık-Küçükkuyu field near Çanakkale province (MTA, 2009; MTA 2010c). Natural radiation sources are found in the volcanic tuffs surfacing between Küçükkuyu and Ayvacık with phosphate nodules (Atabey, 2006). There are magnetite, thorite, zircon and titan minerals in the sands at the Geyikli beach close to the region with uranium and thorium contents in geochemical analysis, and the minerals that cause radioactivity are in the form of thorite and uraninites (Andaç, 1971). The origin of these radioactive minerals are the granitic rocks surfacing around Geyikli (Andaç, 1971). It has been determined that the radiated sands in the vicinity of Aladag village in Ezine district are granitoidic rocks and they are rich in $\mathrm{U}$ and Th (Yılmaz-Şahin et al., 2004, 2006; Örgün et al., 2005; Atabey, 2008a; Atabey and Ünal, 2008). Günaydin (2017) stated that U enrichment in and around Arkkl is related to phosphate, that phosphates in this region are sedimentary and are of hydrothermal type associated with fault lines. The uranium minerals observed in the X-Ray Diffractometers analysis (XRD) of the fault lines are in the composition of bayleite and ningyoite in the old works of MTA (Günaydın, 2017). High natural radiation values were reached in the fault zones near Örencik and Feyzullah Tepe in the northwest of Arıklı (Atabey, 2008a). Magnesite breccias located in the northwest of Arikl1 were formed by the effect of hydrothermal solutions and there are up to $700 \mathrm{ppm}$ of $U$ anomalies and greater than $1000 \mathrm{ppm}$ of Th anomalies in this fault zone (Günaydın, 2017). Fluid inclusion studies have not yet been carried out for mineralization in fault zones developing due to the opening tectonics in the region. In this paper we put forward for the first time the microthermometric data obtained from magnesites observed in these fault zones will also shed light on uranium and thorium mineralization.

\section{Material and method}

It is aimed to determine the relationship between the discontinuity structures of U-Th enrichments, which were previously detected around Arıklı region, to determine the $\mathrm{U}$ and $\mathrm{Th}$ values in this discontinuity, and to reveal the microthermometric properties of hydrothermal magnesites in the fault lines with high $\mathrm{U}$ and $\mathrm{Th}$ values.

\subsection{Analyses}

\subsubsection{Geochemical analysis}

Geochemical analyzes were carried out at the Istanbul Technical University Geochemistry Research Laboratory (ITU-JAL). Major oxides were determined by XRF (X-Ray Floresance) method in BRUKER S8 TIGER device, and trace elements were determined by ICP-MS (Inductively Coupled Plasma Mass Spectroscopy) method in PerkinElmer ELAN 6000 DRC-e device.

\subsubsection{Fluid inclusion studies}

Microthermometric measurements from magnesite related to mineralization were made at ITU JAL using Leica DFC-320, Leica DM 2500, Nikon SMZ800N microscopes integrated into Linkam THMG-600 heating-cooling system. 5 samples were prepared for fluid inclusion study from magnesites located in the fault zone associated with U-Th enrichment in the study area. Among the primary inclusions observed in these samples, Eutectic Temperature $\left(\mathrm{Te}^{\circ} \mathrm{C}\right)$, Final Ice Melting Temperatures $\left(\mathrm{Tm}-\right.$ ice $\left.^{\circ} \mathrm{C}\right)$ and Homogenization Temperature $\left(\mathrm{Th}^{\circ} \mathrm{C}\right)$ were measured.

\section{Regional geology}

The study area is located in Northwest Anatolia, within the boundaries of Biga Peninsula, east of Ayvacık (Çanakkale) district. The study area is located in the Sakarya zone as a geodynamic location (Figure 1.1a). Okay et al., (1990) defined the units in Çanakkale region as pre-Tertiary and post-Tertiary units. In their subsequent studies, it was divided into three pre-Tertiary tectonic zones observed in NE-SW direction (Okay et al., 2001, Okay and Altıner, 2004). These zones are from 
northeast to southwest; Ezine Zone, AyvackkKarabiga Zone and Sakarya Zone.

There are many metamorphic facies along with ophiolitic rock groups, magmatic, volcanic and sedimentary rock groups located in the north of
Edremit Bay (Figure 1.1b) (Okay and Satır, 2000a; Şengün et al., 2011). In the vicinity of Arıkl, the Miocene Pliocene aged continental sediments and Cretaceous aged Çetmi melange are outcrop (Figure 1.1b).

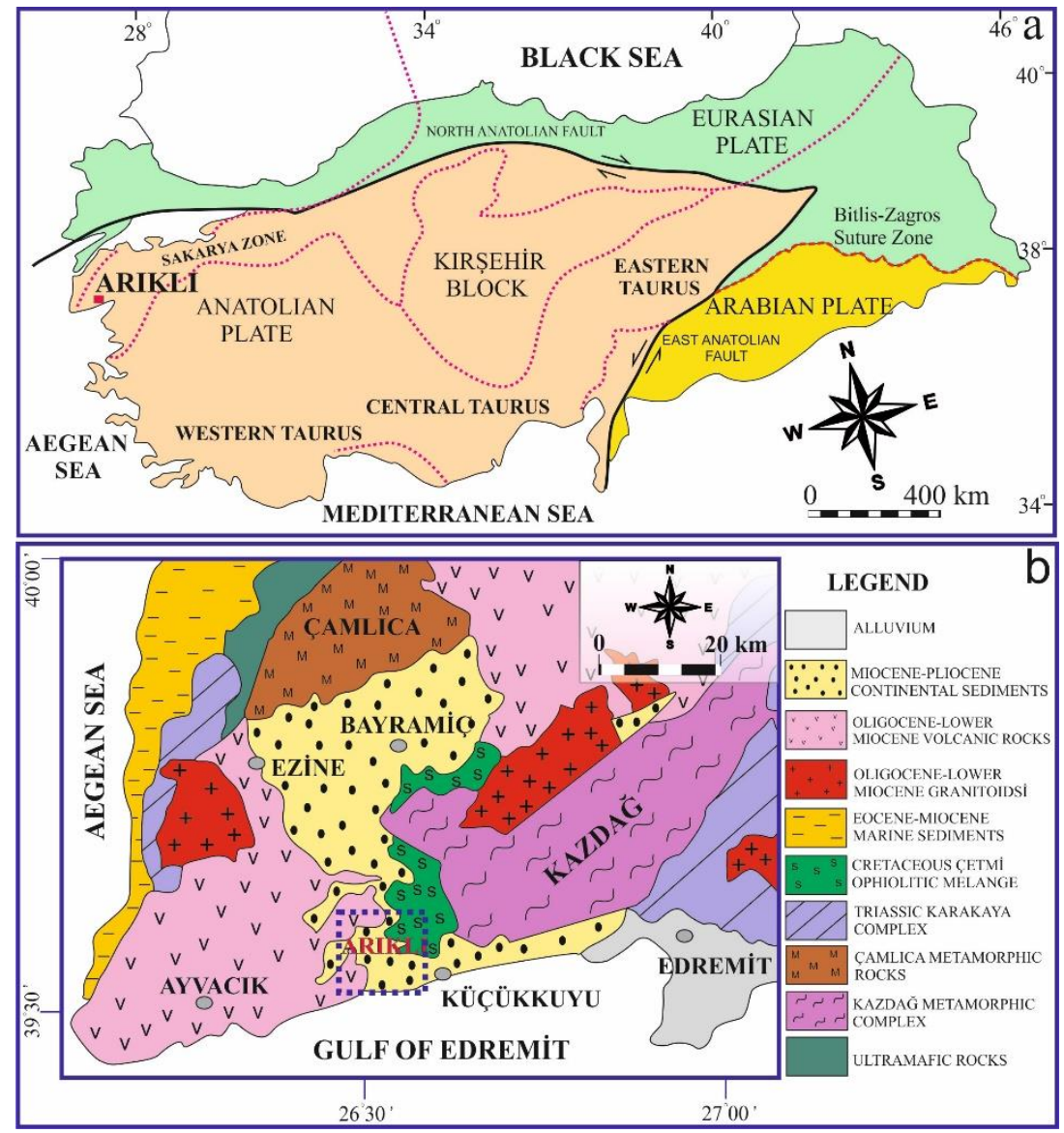

Figure 1. a. Tectonic location of the study area (modified from Iş1k, 2016), b. Generalized geology map of the Biga region and location of the study area (modified from Okay and Satır, 2000a; Şengün et al., 2011).

\subsection{Geology of the study area}

The Cretaceous Aged Çetmi Ophiolitic Melange, Küçükkuyu Formation and Quaternary aged alluvial deposits are located in the study area from older to younger. In the Küçükkuyu formation, shale-sandstone member, and Arikli tuff member were distinguished and diabases that cut these units were mapped on the Örencik Tepe (Figure 2).

\subsection{1. Çetmi ophiolitic melange}

The oldest unit of the study area is Çetmi Ophiolitic Melange (Siyako et al., 1989; Okay et al., 1990). This unit, which spreads around Çamurlu Tepe, Sivri Tepe and Hidırkaya Tepe in the northeast of the study area, consists mainly of spilitic mafic volcanic rocks, pyroclastic rocks, limestone, chertradiolarite, shale and graywacke. In addition, it is located in different levels of serpentinite, radiolaria chert and garnet micaschist, metabasic rocks that have undergone metamorphism in eclogite facies (Günaydın, 2017).

Çetmi melange consists of mafic volcanic rocks, limestones, radiolarite-chert, intercalation of graywacke and shale contain serpentinite blocks and metamorphic rocks consisting of tectonic slices (Okay and Satır, 2000b; Beccaletto and Jenny, 2004).

Beccaletto et al., (2005) stated that the melange in the region was affected by a severe tension during the Paleogene period. The contacts of the units of the ophiolitic melange are limited by the E-W and NE-SW trending dip-slip faults (Figure 2). 


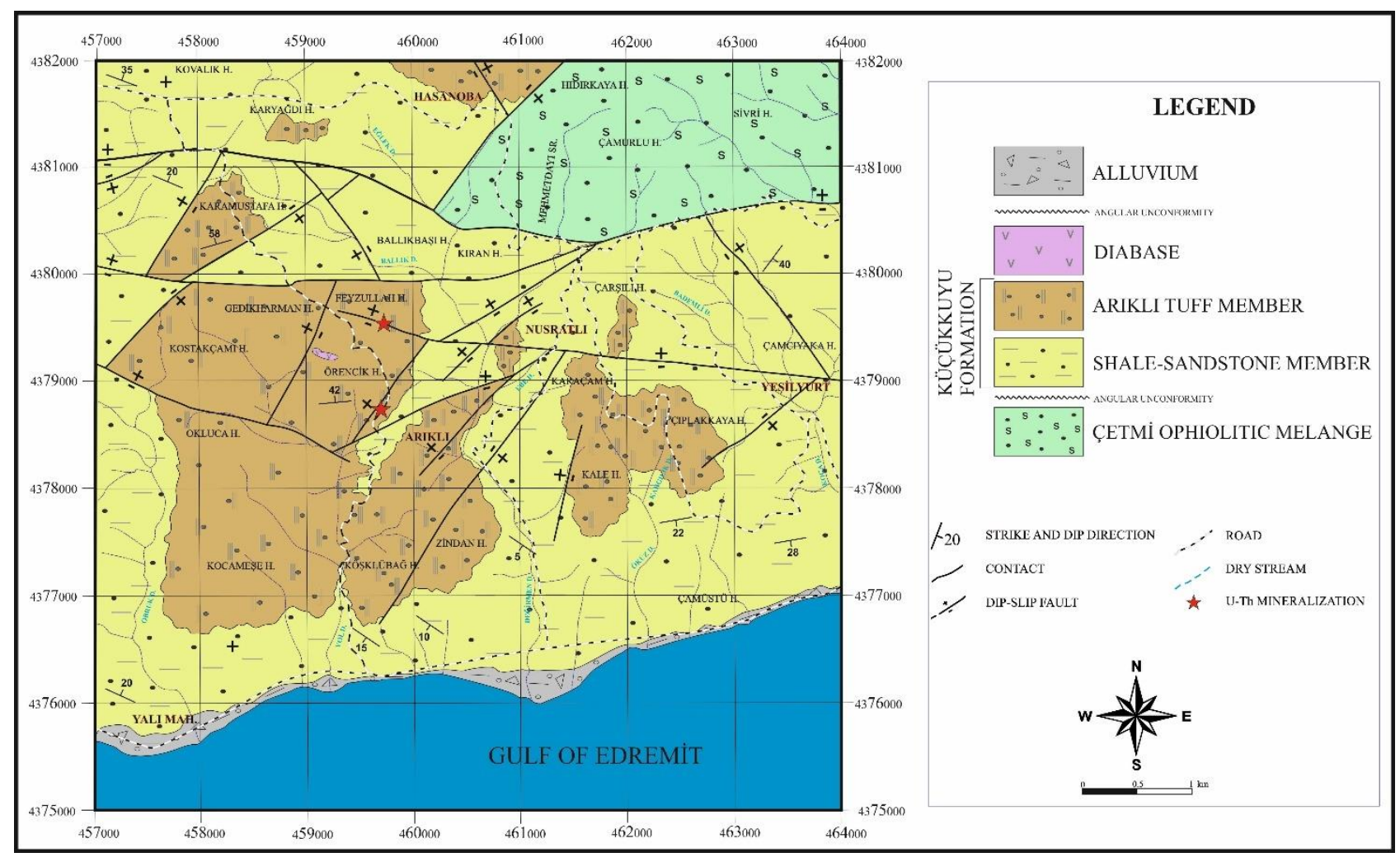

Figure 2. Geological map of the study area.

\subsubsection{Küçükkuyu formation}

This unit, which was named for the first time by Saka (1979), includes continental sediments formed simultaneously with Oligo-Miocene volcanism, tuff and pyroclastic rocks of this volcanism (Siyako et al., 1989). The base of the unit consists of conglomerate and towards the upper levels, it passes into sandstone, mudstone, marl, claystone, shale and tuffs. For this reason, mapping was carried out seperately as a member of the continental clastics and a member of Arıklı ignimbrites. Some researchers have determined the age of the formation as Lower-Middle Miocene (İnci, 1984; Kesgin, 2001; Çiftçi et al., 2004).

\section{Shale-sandstone member}

This unit, which outcrops in most of the study area, consists of shale-sandstone intercalation. The unit has lateral and vertical transition with Arıkl1 ignimbrites, and located side by side with Cretaceous aged Çetmi ophiolitic melange by dipslip fault in the northeast. In the south, this unit is covered with angular unconformity by Quaternary aged alluvial deposit (Figure 2).

\section{Arlklı tuff member}

Arıkl iginmbrites are widely outcropped in the study area around Arıklı (Figure 2). This unit, which is observed topographically at higher levels, spreads in Örencik Tepe, Feyzullah Tepe, west of Nusratl1, around Kale Tepe and Hasanoba region. Arıklı ignimbirites, consists of andesite-dacite lava and tuffs (Dönmez et al., 2005). Günaydın (2017) divided the tuffs into three groups: vitric, crystal and lithic tuff. In the northwest of Arıklı, there are hydrothermal magnesite breccias in dip-slip fault contacts (Günaydın, 2017). The contact relationship of this unit, which has a lateral vertical transition with the shale-sandstone member, is tectonic in some regions. This unit is located side by side with Çetmi ophiolitic melange by dip-slip fault in the north of Hasanoba. In the north of Örencik Tepe, diabase dyke cuts this unit with an E-W trending (Figure 2).

\section{Diabase}

This unit, which is observed in limited areas in the north of Örencik Tepe in the study area, cuts the ignimbrites with an E-W trending.

\section{Ore geochemistry}

Günaydın (2017) stated that U-Th mineralization in this region is of hydrothermal type associated with fault lines. Since $U$ and Th mineralization is not visible, samples were collected from ignimbrites and fault zones around Arrklı and Nusratlı. Analysis results of 48 compiled samples are given 
in Table 1. According to the results of the analysis, $\mathrm{U}$ and Th anomalies are observed in the NE-SW and NW-SE dip-slip fault zones observed in the north west of Arkkl1 (Figure 3). The highest $U$ and Th values are found in the area where magnesite breccias in the northwest of Ar1kl1 (Figure 4). In the F35A and F35B samples, anomalies of $U$ are between 64-1640 ppm and Th anomalies are between 302-11813 ppm (Table 1). In samples compiled around Örencik Tepe and Feyzullah Tepe (F36, F37, F39, F40A, F40B, F41, F41A, F42A, F42B) $U$ are between $0-11 \mathrm{ppm}$ and Th are between 21-397 ppm (Table 1). As a result of these data, $U$ and Th mineralizations in the region are related to fault zones developing in Ar1kl1 ignimbrites.
Günaydin (2017) stated that the presence of minerals such as barite and magnesite in the fault lines are of hydrothermal origin of mineralization.

Besides these, bestowing to the geochemical analysis of the samples gathered in the region, in $\mathrm{U}$ and $\mathrm{Th}$ rich specimens, $\mathrm{MgO}$ is between 17.23$40.10 \%, \mathrm{CaO} 3.08-30.57 \%$ and $\mathrm{SiO}_{2}$ is between 2$28.69 \%$ (Table 1). U-Th anomaly was not detected in many samples gathered from fault zones. Although Günaydin (2017) determined $\mathrm{P}_{2} \mathrm{O}_{5}$ between $1.7 \%$ and $32.4 \%$ in his analysis in the $\mathrm{U}$ and Th rich zone (Feyzullah and Örencik Hill), $\mathrm{P}_{2} \mathrm{O}_{5}$ contents were between $0.03-0.93 \%$ in the samples solicited in this study.

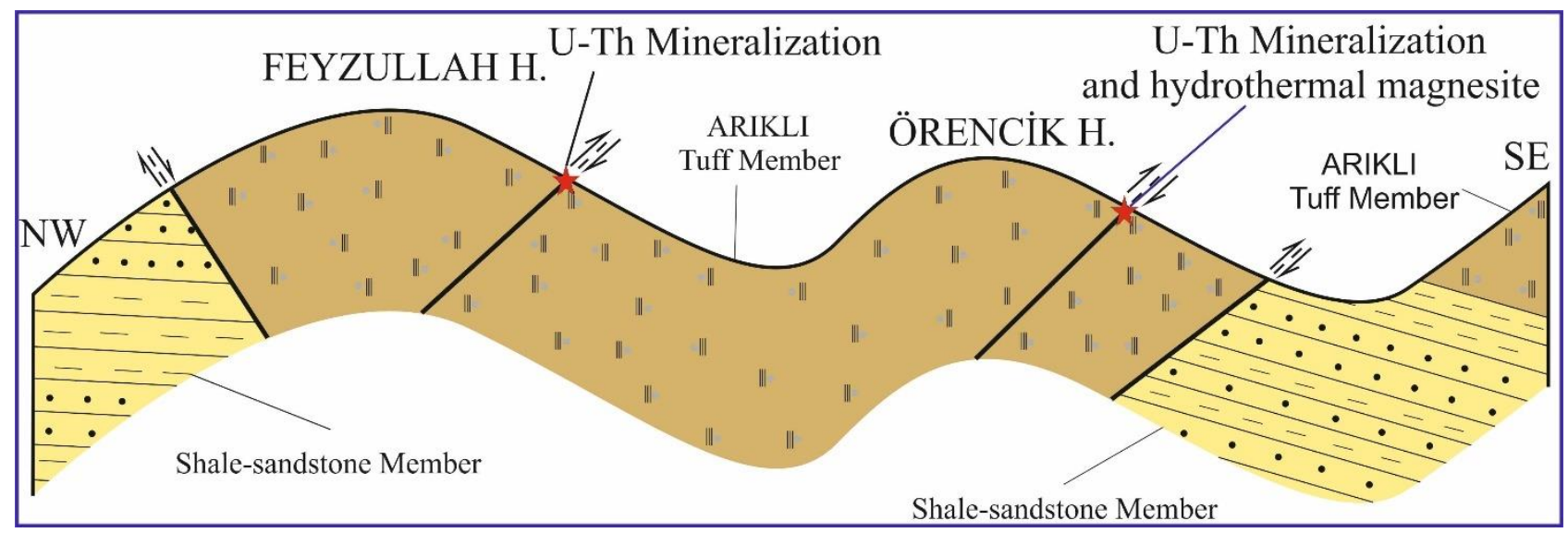

Figure 3. U-Th anomalies observed in fault zones in northeast of Arkklı and general view of the units (without scale).

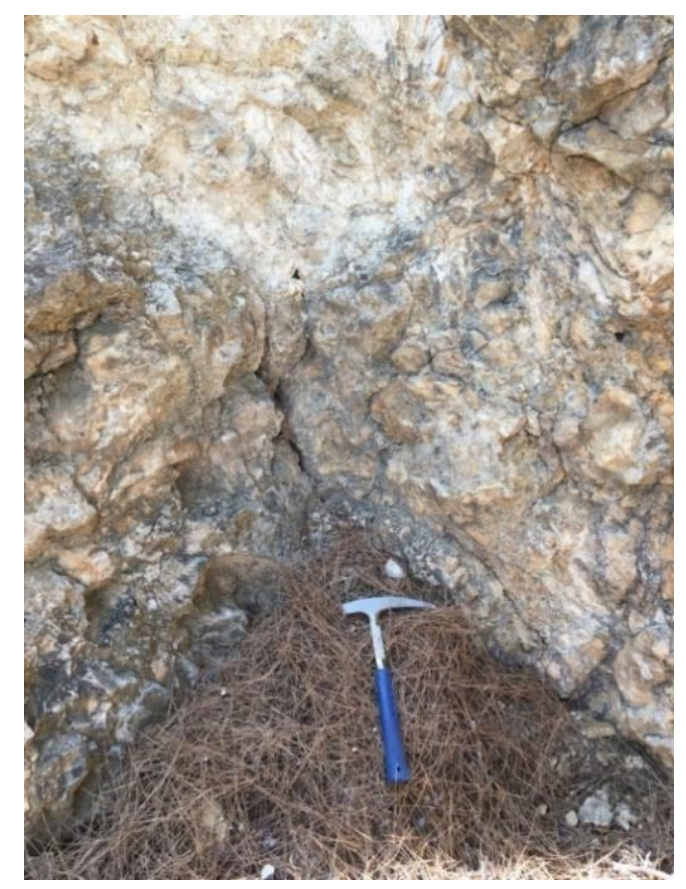

Figure 4. General view of hydrothermal magnesites in northwest of Arıkl1. 
Table 1. Major oxide (\%) and trace element (ppm) values of the samples compiled in the study area.

\begin{tabular}{|c|c|c|c|c|c|c|c|c|c|c|c|c|}
\hline Major Oxide (\%) & F1 & F2_3 & F4 & F5 & F6 & F7 & F9A & F9B & F10 & F11A & F11B & F12 \\
\hline $\mathrm{SiO}_{2}$ & 63.09 & 61.52 & 38.33 & 60.54 & 64.17 & 71.32 & 63.64 & 61.59 & 73.9 & 55.51 & 59.25 & 60.12 \\
\hline $\mathrm{Al}_{2} \mathrm{O}_{3}$ & 15.83 & 15.07 & 10.09 & 14.89 & 10.55 & 13.17 & 15.39 & 12.89 & 11.13 & 19.96 & 18.55 & 15.9 \\
\hline $\mathrm{Fe}_{2} \mathrm{O}_{3}$ & 3.17 & 2.07 & 4.43 & 7.08 & 3.26 & 3.85 & 6.47 & 5.32 & 2.99 & 6.52 & 5.13 & 4.75 \\
\hline $\mathrm{MgO}$ & 0.77 & 1.26 & 5.82 & 0.4 & 2.27 & 0.4 & 0.44 & 0.5 & 0.46 & 0.84 & 0.79 & 0.13 \\
\hline $\mathrm{CaO}$ & 0.93 & 2.93 & 15.31 & 0.2 & 5.6 & 0.17 & 0.14 & 0.79 & 0.08 & 0.66 & 0.68 & 0.36 \\
\hline $\mathrm{Na}_{2} \mathrm{O}$ & 0.31 & 0.1 & 0.19 & 0.23 & 0.14 & 0.14 & 0.12 & 0.19 & 0.13 & 0.85 & 0.71 & 0.09 \\
\hline $\mathrm{K}_{2} \mathrm{O}$ & 9.87 & 8.93 & 7.43 & 13.19 & 5 & 7.64 & 9.72 & 6.98 & 8.73 & 8.81 & 8.13 & 15.4 \\
\hline $\mathrm{TiO}_{2}$ & 0.45 & 0.38 & 0.49 & 0.75 & 0.45 & 0.7 & 0.95 & 0.78 & 0.46 & 0.95 & 0.87 & 0.91 \\
\hline $\mathrm{P}_{2} \mathrm{O}_{5}$ & 0.09 & 0.09 & 0.11 & 0.2 & 0.58 & 0.21 & 0.41 & 0.3 & 0.07 & 0.1 & 0.11 & 0.36 \\
\hline $\mathrm{MnO}$ & 0.07 & 0.08 & 0.17 & 0.11 & 0.08 & 0.07 & 0.17 & 0.14 & 0.02 & 0.02 & 0.01 & 0.11 \\
\hline $\mathrm{Cr}_{2} \mathrm{O}_{3}$ & 0.39 & 0.23 & 0.01 & 0.04 & 0.38 & 0.34 & 0.05 & 0.07 & 0.45 & 0.28 & 0 & 0 \\
\hline $\mathrm{SO}_{3}$ & 0.38 & 0.36 & 0.06 & 0.02 & 0.06 & 0.03 & 0.02 & 0.10 & 0.03 & 0.49 & 0.60 & 0.03 \\
\hline LOI & 4.03 & 6.63 & 17.34 & 2.17 & 7.25 & 1.80 & 2.21 & 10.15 & 1.43 & 4.78 & 4.94 & 1.38 \\
\hline TOTAL & 99.47 & 99.63 & 99.88 & 99.90 & 99.85 & 99.91 & 99.79 & 99.83 & 99.47 & 99.90 & 99.91 & 99.84 \\
\hline Trace Element (ppm) & F1 & F2_3 & $\mathbf{F 4}$ & F5 & F6 & F7 & F9A & F9B & F10 & F11A & F11B & F12 \\
\hline As & 357 & 315 & 312 & 261 & 150 & 241 & 295 & 249 & 85 & 141 & 157 & 682 \\
\hline $\mathrm{Ba}$ & 1172 & 1117 & 612 & 451 & 300 & 412 & 328 & 389 & 248 & 1013 & 1039 & 756 \\
\hline $\mathrm{Br}$ & ND & ND & ND & ND & ND & ND & ND & 15 & ND & ND & ND & ND \\
\hline $\mathrm{Ce}$ & 375 & 106 & 60 & ND & ND & ND & 391 & 339 & ND & ND & ND & 1491 \\
\hline $\mathrm{Cl}$ & 2874 & 580 & 118 & ND & 153 & ND & ND & 130 & 137 & 88 & 106 & 110 \\
\hline Co & ND & ND & ND & ND & ND & ND & 12 & 3 & 4 & ND & ND & ND \\
\hline $\mathrm{Cu}$ & 41 & 49 & 29 & 31 & 46 & 33 & 48 & 35 & 46 & 42 & ND & 38 \\
\hline $\mathrm{Ga}$ & ND & 39 & 33 & 21 & ND & 27 & ND & ND & ND & ND & 23 & 40 \\
\hline Ge & ND & $\mathrm{ND}$ & 15 & ND & ND & ND & ND & ND & ND & ND & ND & ND \\
\hline Mo & ND & ND & ND & ND & 60 & ND & ND & ND & ND & ND & ND & ND \\
\hline $\mathrm{Nb}$ & 17 & 14 & ND & 13 & 11 & 9 & 16 & ND & ND & 10 & 10 & ND \\
\hline $\mathrm{Ni}$ & 25 & 35 & 102 & 201 & 210 & 278 & 409 & 225 & 111 & ND & 27 & 102 \\
\hline $\mathrm{Pb}$ & 60 & 59 & ND & ND & ND & ND & ND & ND & ND & ND & ND & 54 \\
\hline $\mathrm{Rb}$ & 232 & 197 & 144 & 297 & 123 & 152 & 168 & 132 & 216 & 274 & 236 & 287 \\
\hline $\mathrm{Se}$ & ND & ND & ND & ND & ND & ND & ND & ND & ND & ND & ND & ND \\
\hline $\mathrm{Sr}$ & 116 & 136 & 386 & 53 & 355 & 28 & 24 & 44 & 34 & 177 & 159 & 28 \\
\hline Th & 34 & 71 & ND & ND & ND & ND & ND & ND & ND & ND & ND & ND \\
\hline $\mathrm{U}$ & ND & $\mathrm{ND}$ & ND & ND & ND & ND & $\mathrm{ND}$ & ND & ND & ND & ND & ND \\
\hline V & ND & ND & ND & ND & ND & ND & 154 & ND & ND & ND & ND & ND \\
\hline Y & ND & $\mathrm{ND}$ & ND & ND & 12 & ND & ND & ND & ND & 14 & ND & ND \\
\hline $\mathrm{Yb}$ & ND & $\mathrm{ND}$ & ND & ND & ND & ND & $\mathrm{ND}$ & ND & $\mathrm{ND}$ & ND & ND & ND \\
\hline $\mathrm{Zn}$ & ND & ND & ND & $\mathrm{ND}$ & ND & ND & $\mathrm{ND}$ & ND & 28 & ND & ND & ND \\
\hline $\mathrm{Zr}$ & 348 & 308 & 111 & 178 & 221 & 121 & 172 & 168 & 157 & 202 & 177 & 123 \\
\hline
\end{tabular}


Table 1. Continued.

\begin{tabular}{|c|c|c|c|c|c|c|c|c|c|c|c|c|}
\hline Major Oxide (\%) & F13 & F14 & F22A & F22B & F23 & F24 & F25 & F26 & F27_A & F27_B & F28_A & F28_B \\
\hline $\mathrm{SiO}_{2}$ & 34.96 & 64.58 & 48.51 & 54.01 & 54.75 & 63.24 & 59 & 59.39 & 47.45 & 50.37 & 59.19 & 59.46 \\
\hline $\mathrm{Al}_{2} \mathrm{O}_{3}$ & 9.82 & 14.87 & 14.64 & 14.85 & 14.87 & 9.97 & 13.77 & 14.21 & 11.99 & 12.82 & 13.87 & 13.76 \\
\hline $\mathrm{Fe}_{2} \mathrm{O}_{3}$ & 4.75 & 2.44 & 5.48 & 5.7 & 5.99 & 3.02 & 4.08 & 3.95 & 6.26 & 6.42 & 6.07 & 5.89 \\
\hline $\mathrm{MgO}$ & 2.08 & 0.72 & 7.07 & 4.92 & 4.54 & 3.53 & 3.12 & 3.05 & 4.4 & 2.97 & 3.23 & 3.12 \\
\hline $\mathrm{CaO}$ & 26.18 & 0.56 & 9.72 & 4.36 & 3.68 & 6.78 & 4.68 & 4.34 & 10.46 & 11.4 & 3.63 & 3.61 \\
\hline $\mathrm{Na}_{2} \mathrm{O}$ & 1.67 & 0.19 & 2.24 & 1.15 & 1.26 & 2.08 & 1.5 & 1.65 & 1.34 & 0.95 & 0.97 & 0.98 \\
\hline $\mathrm{K}_{2} \mathrm{O}$ & 1.25 & 13.67 & 3.5 & 5.96 & 5.8 & 3.19 & 6.34 & 6.51 & 4 & 3.36 & 3.93 & 3.9 \\
\hline $\mathrm{TiO}_{2}$ & 0.47 & 0.38 & 0.95 & 0.83 & 0.81 & 0.48 & 0.63 & 0.63 & 0.7 & 0.66 & 0.78 & 0.79 \\
\hline $\mathrm{P}_{2} \mathrm{O}_{5}$ & 0.16 & 0.07 & 0.31 & 0.24 & 0.23 & 0.17 & 0.21 & 0.22 & 0.13 & 0.17 & 0.25 & 0.26 \\
\hline $\mathrm{MnO}$ & 0.08 & 0.04 & 0.07 & 0.12 & 0.1 & 0.06 & 0.07 & 0.1 & 0.17 & 0.12 & 0.13 & 0.13 \\
\hline $\mathrm{Cr}_{2} \mathrm{O}_{3}$ & 0.53 & 0.3 & 0.01 & 0.03 & 0.29 & 0.26 & 0.01 & 0 & 0.01 & 0.05 & 0.05 & 0.05 \\
\hline $\mathrm{SO}_{3}$ & 0.31 & 0.04 & 0.09 & 0.15 & 0.14 & 0.10 & 0.15 & 0.14 & 0.22 & 0.19 & 0.12 & 0.13 \\
\hline LOI & 17.44 & 1.87 & 6.99 & 7.32 & 7.15 & 6.61 & 6.01 & 5.37 & 12.69 & 10.19 & 7.58 & 7.70 \\
\hline TOTAL & 99.83 & 99.84 & 99.75 & 99.80 & 99.50 & 99.87 & 99.82 & 99.81 & 99.88 & 99.86 & 99.89 & 99.88 \\
\hline Trace Element (ppm) & F13 & F14 & F22A & F22B & F23 & F24 & F25 & F26 & F27_A & F27_B & F28_A & F28_B \\
\hline As & 32 & 148 & 107 & 182 & 193 & 106 & 213 & 1942 & 132 & 110 & 107 & 90 \\
\hline $\mathrm{Ba}$ & 1084 & 548 & 1370 & 1269 & 1157 & 739 & 1784 & 111 & 457 & 1733 & 848 & 862 \\
\hline $\mathrm{Br}$ & ND & ND & ND & $\mathrm{ND}$ & ND & ND & ND & $\mathrm{ND}$ & ND & ND & ND & ND \\
\hline $\mathrm{Ce}$ & ND & 403 & ND & $\mathrm{ND}$ & 230 & $\mathrm{ND}$ & 485 & $\mathrm{ND}$ & ND & ND & ND & ND \\
\hline $\mathrm{Cl}$ & 277 & ND & 164 & 124 & 273 & 2885 & 142 & 156 & $\mathrm{ND}$ & ND & ND & ND \\
\hline Co & ND & ND & ND & ND & ND & ND & 6 & 9 & 4 & ND & 3 & ND \\
\hline $\mathrm{Cu}$ & 44 & 22 & 94 & 58 & 68 & 36 & 51 & 54 & 62 & 43 & 44 & 50 \\
\hline $\mathrm{Ga}$ & ND & 71 & 58 & 30 & 37 & ND & 26 & 25 & ND & ND & 15 & ND \\
\hline $\mathrm{Ge}$ & ND & 18 & ND & 21 & ND & ND & ND & $\mathrm{ND}$ & ND & ND & ND & ND \\
\hline Mo & ND & ND & $\mathrm{ND}$ & $\mathrm{ND}$ & ND & $\mathrm{ND}$ & $\mathrm{ND}$ & $\mathrm{ND}$ & $\mathrm{ND}$ & $\mathrm{ND}$ & ND & ND \\
\hline $\mathrm{Nb}$ & ND & 20 & ND & ND & 8 & ND & 11 & ND & ND & ND & $\mathrm{ND}$ & 12 \\
\hline $\mathrm{Ni}$ & 58 & 32 & 60 & 191 & 202 & 45 & 51 & 40 & 83 & 119 & 173 & 166 \\
\hline $\mathrm{Pb}$ & 50 & 59 & $\mathrm{ND}$ & 42 & ND & ND & 37 & 44 & ND & ND & $\mathrm{ND}$ & 35 \\
\hline $\mathrm{Rb}$ & 29 & 375 & 93 & 160 & 186 & 73 & 159 & 167 & 110 & 106 & 115 & 112 \\
\hline $\mathrm{Se}$ & ND & ND & $\mathrm{ND}$ & ND & ND & ND & $\mathrm{ND}$ & ND & $\mathrm{ND}$ & $\mathrm{ND}$ & $\mathrm{ND}$ & $\mathrm{ND}$ \\
\hline $\mathrm{Sr}$ & 670 & 30 & 1033 & 397 & 439 & 433 & 421 & 500 & 443 & 419 & 268 & 273 \\
\hline Th & ND & 46 & ND & ND & ND & ND & ND & ND & $\mathrm{ND}$ & ND & ND & ND \\
\hline $\mathrm{U}$ & ND & ND & $\mathrm{ND}$ & $\mathrm{ND}$ & ND & ND & ND & $\mathrm{ND}$ & ND & $\mathrm{ND}$ & ND & ND \\
\hline V & 38 & 0 & 168 & 165 & 0 & 0 & ND & ND & ND & ND & 145 & 153 \\
\hline $\mathrm{Y}$ & 0 & 0 & 0 & 0 & 11 & 0 & ND & $\mathrm{ND}$ & ND & ND & ND & ND \\
\hline $\mathrm{Yb}$ & 0 & 0 & 0 & 0 & 0 & 74 & ND & ND & ND & ND & ND & ND \\
\hline $\mathrm{Zn}$ & 0 & 0 & 0 & 0 & 0 & 0 & ND & ND & ND & ND & ND & ND \\
\hline $\mathrm{Zr}$ & 286 & 441 & 304 & 219 & 375 & 228 & 266 & 386 & 222 & 248 & 179 & 188 \\
\hline
\end{tabular}


Table 1. Continued.

\begin{tabular}{|c|c|c|c|c|c|c|c|c|c|c|c|c|}
\hline Major Oxide (\%) & F29A & F29B & F30 & F31_A & F31_B & F35_A & F35_B & F36 & F37 & F39 & F40_A & F40_B \\
\hline $\mathrm{SiO}_{2}$ & 46.88 & 51.94 & 57.76 & 60.46 & 59.9 & 2 & 4.64 & 7.75 & 6.1 & 6.1 & 3.66 & 28.69 \\
\hline $\mathrm{Al}_{2} \mathrm{O}_{3}$ & 12.16 & 15.07 & 11.29 & 12.1 & 12.46 & 0.31 & 0.83 & 1.99 & 0.53 & 1.26 & 0.98 & 8.13 \\
\hline $\mathrm{Fe}_{2} \mathrm{O}_{3}$ & 6.1 & 6.39 & 4.93 & 5.34 & 5.2 & 1.01 & 0.22 & 0.55 & 0.47 & 0.23 & 0.39 & 1.85 \\
\hline $\mathrm{MgO}$ & 5.47 & 3.79 & 5.04 & 5.21 & 4.95 & 17.25 & 40.1 & 36.74 & 17.69 & 40.09 & 33.66 & 17.23 \\
\hline $\mathrm{CaO}$ & 9.32 & 5.51 & 7.18 & 3.87 & 3.98 & 30.57 & 4.93 & 4.58 & 29.27 & 3.08 & 12.51 & 9.94 \\
\hline $\mathrm{Na}_{2} \mathrm{O}$ & 1.69 & 1.49 & 1.32 & 1.18 & 1.29 & 0 & 0.22 & 0 & 0 & 0 & 0 & 0.09 \\
\hline $\mathrm{K}_{2} \mathrm{O}$ & 3.5 & 5.24 & 3.7 & 3.95 & 3.98 & 0.03 & 0.04 & 1.25 & 0.09 & 0.03 & 0.24 & 1.86 \\
\hline $\mathrm{TiO}_{2}$ & 0.67 & 0.72 & 0.64 & 0.69 & 0.68 & 0.1 & 0.02 & 0.05 & 0.02 & 0.04 & 0.04 & 0.25 \\
\hline $\mathrm{P}_{2} \mathrm{O}_{5}$ & 0.17 & 0.17 & 0.17 & 0.18 & 0.17 & 0.93 & 0.05 & 0.03 & 0.04 & 0.03 & 0.04 & 0.13 \\
\hline $\mathrm{MnO}$ & 0.18 & 0.1 & 0.09 & 0.09 & 0.09 & 0.05 & 0.02 & 0.02 & 0.04 & 0.02 & 0.04 & 0.05 \\
\hline $\mathrm{Cr}_{2} \mathrm{O}_{3}$ & 0.03 & 0.02 & 0.08 & 0.08 & 0.08 & 0 & 0 & 0 & 0 & 0 & 0 & 0 \\
\hline $\mathrm{SO}_{3}$ & 0.52 & 0.39 & 0.23 & 0.13 & 0.13 & 0.03 & 0.01 & 0.01 & 0.02 & 0.01 & 0.01 & 0.06 \\
\hline LOI & 13.00 & 8.41 & 7.31 & 6.46 & 6.84 & 45.57 & 48.79 & 46.94 & 45.57 & 49.02 & 48.24 & 31.51 \\
\hline TOTAL & 99.82 & 99.77 & 99.87 & 99.84 & 99.78 & 97.95 & 99.89 & 99.95 & 99.98 & 99.99 & 99.94 & 99.85 \\
\hline Trace Element (ppm) & F29A & F29B & F30 & F31_A & F31_B & F35_A & F35_B & F36 & F37 & F39 & F40_A & F40_B \\
\hline As & 207 & 183 & 94 & 93 & 93 & 149 & 30 & 164 & 69 & 16 & 80 & 250 \\
\hline $\mathrm{Ba}$ & 958 & 5039 & 1122 & 869 & 869 & 765 & $\mathrm{ND}$ & $\mathrm{ND}$ & $\mathrm{ND}$ & $\mathrm{ND}$ & $\mathrm{ND}$ & 304 \\
\hline $\mathrm{Br}$ & $\mathrm{ND}$ & ND & ND & ND & ND & ND & $\mathrm{ND}$ & ND & $\mathrm{ND}$ & $\mathrm{ND}$ & ND & ND \\
\hline $\mathrm{Ce}$ & $\mathrm{ND}$ & $\mathrm{ND}$ & $\mathrm{ND}$ & ND & ND & ND & $\mathrm{ND}$ & $\mathrm{ND}$ & $\mathrm{ND}$ & $\mathrm{ND}$ & ND & $\mathrm{ND}$ \\
\hline $\mathrm{Cl}$ & 157 & 148 & ND & ND & 110 & ND & 140 & 115 & ND & 95 & 137 & ND \\
\hline Co & ND & ND & ND & 6 & 7 & 30 & $\mathrm{ND}$ & ND & $\mathrm{ND}$ & $\mathrm{ND}$ & ND & ND \\
\hline $\mathrm{Cu}$ & 46 & 60 & 39 & 46 & 38 & ND & 18 & 22 & 24 & $\mathrm{ND}$ & 20 & 32 \\
\hline $\mathrm{Ga}$ & 18 & 19 & 18 & 17 & ND & ND & $\mathrm{ND}$ & $\mathrm{ND}$ & $\mathrm{ND}$ & $\mathrm{ND}$ & ND & ND \\
\hline $\mathrm{Ge}$ & $\mathrm{ND}$ & $\mathrm{ND}$ & $\mathrm{ND}$ & $\mathrm{ND}$ & ND & $\mathrm{ND}$ & $\mathrm{ND}$ & $\mathrm{ND}$ & $\mathrm{ND}$ & $\mathrm{ND}$ & ND & ND \\
\hline Mo & $\mathrm{ND}$ & $\mathrm{ND}$ & $\mathrm{ND}$ & $\mathrm{ND}$ & $\mathrm{ND}$ & $\mathrm{ND}$ & $\mathrm{ND}$ & $\mathrm{ND}$ & $\mathrm{ND}$ & $\mathrm{ND}$ & $\mathrm{ND}$ & $\mathrm{ND}$ \\
\hline $\mathrm{Nb}$ & 7 & 9 & 7 & 8 & $\mathrm{ND}$ & 62 & $\mathrm{ND}$ & $\mathrm{ND}$ & $\mathrm{ND}$ & $\mathrm{ND}$ & $\mathrm{ND}$ & $\mathrm{ND}$ \\
\hline $\mathrm{Ni}$ & 183 & 159 & 198 & 231 & 226 & 24 & 12 & 17 & $\mathrm{ND}$ & 20 & 13 & 34 \\
\hline $\mathrm{Pb}$ & 56 & $\mathrm{ND}$ & $\mathrm{ND}$ & ND & ND & 43 & $\mathrm{ND}$ & $\mathrm{ND}$ & $\mathrm{ND}$ & $\mathrm{ND}$ & ND & 28 \\
\hline $\mathrm{Rb}$ & 127 & 174 & 94 & 112 & 112 & 44 & ND & 25 & ND & ND & ND & 54 \\
\hline $\mathrm{Se}$ & ND & ND & ND & ND & ND & ND & ND & ND & ND & $\mathrm{ND}$ & ND & ND \\
\hline $\mathrm{Sr}$ & 540 & 475 & 454 & 358 & 363 & 2977 & 471 & 434 & 1175 & 437 & 978 & 485 \\
\hline Th & $\mathrm{ND}$ & ND & ND & ND & ND & 11813 & 302 & 21 & 133 & 225 & 261 & 397 \\
\hline $\mathrm{U}$ & ND & ND & ND & ND & ND & 1640 & 64 & $\mathrm{ND}$ & $\mathrm{ND}$ & 11 & $\mathrm{ND}$ & $\mathrm{ND}$ \\
\hline $\mathrm{V}$ & ND & $\mathrm{ND}$ & $\mathrm{ND}$ & 142 & ND & ND & $\mathrm{ND}$ & $\mathrm{ND}$ & $\mathrm{ND}$ & $\mathrm{ND}$ & $\mathrm{ND}$ & $\mathrm{ND}$ \\
\hline $\mathrm{Y}$ & 16 & $\mathrm{ND}$ & 18 & $\mathrm{ND}$ & $\mathrm{ND}$ & 864 & $\mathrm{ND}$ & $\mathrm{ND}$ & $\mathrm{ND}$ & $\mathrm{ND}$ & $\mathrm{ND}$ & $\mathrm{ND}$ \\
\hline $\mathrm{Yb}$ & ND & ND & ND & ND & ND & 243 & $\mathrm{ND}$ & ND & $\mathrm{ND}$ & $\mathrm{ND}$ & ND & ND \\
\hline $\mathrm{Zn}$ & $\mathrm{ND}$ & $\mathrm{ND}$ & $\mathrm{ND}$ & 66 & 75 & $\mathrm{ND}$ & $\mathrm{ND}$ & $\mathrm{ND}$ & $\mathrm{ND}$ & $\mathrm{ND}$ & 12 & 41 \\
\hline $\mathrm{Zr}$ & 308 & 304 & 256 & 172 & 175 & ND & 5 & 13 & 30 & 9 & 23 & 232 \\
\hline
\end{tabular}


Table 1. Continued.

\begin{tabular}{|c|c|c|c|c|c|c|c|c|c|c|c|c|}
\hline Major Oxide E (\%) & F41 & F41_A & F42_A & F42_B & $\mathbf{F 4 5}$ & F46_47 & F48 & F49 & F50 & F52 & F53 & F54 \\
\hline $\mathrm{SiO}_{2}$ & 3.02 & 22.12 & 18.56 & 24.34 & 52.26 & 67.21 & 62.92 & 77.51 & 95.7 & 64.49 & 64.16 & 59.53 \\
\hline $\mathrm{Al}_{2} \mathrm{O}_{3}$ & 0.11 & 6.82 & 3.48 & 5.93 & 15.74 & 14.31 & 15.32 & 17.45 & 1.58 & 17.67 & 15.94 & 13.11 \\
\hline $\mathrm{Fe}_{2} \mathrm{O}_{3}$ & 0.31 & 1.13 & 0.83 & 1.03 & 11.27 & 2.9 & 3.94 & 1.22 & 0.77 & 2.07 & 2.55 & 5.92 \\
\hline $\mathrm{MgO}$ & 37.38 & 21.53 & 31.99 & 26.72 & 1.96 & ND & 0.07 & 0.46 & 0.11 & 0.77 & 0.82 & 4.33 \\
\hline $\mathrm{CaO}$ & 10.63 & 7.78 & 1.92 & 2.26 & 1.67 & $\mathrm{ND}$ & 0.16 & 0.13 & 0.32 & 2.03 & 2.39 & 2.75 \\
\hline $\mathrm{Na}_{2} \mathrm{O}$ & ND & ND & 0.64 & 0.45 & 3.91 & 0.07 & 0.36 & ND & ND & 0.21 & 0.24 & 0.74 \\
\hline $\mathrm{K}_{2} \mathrm{O}$ & ND & 0.17 & 0.07 & 0.32 & 6.29 & 13.25 & 14.52 & 3.01 & 0.36 & 5.49 & 6.75 & 5.01 \\
\hline $\mathrm{TiO}_{2}$ & 0.01 & 0.17 & 0.09 & 0.16 & 1.28 & 0.37 & 0.64 & 0.06 & 0.05 & 0.29 & 0.28 & 0.69 \\
\hline $\mathrm{P}_{2} \mathrm{O}_{5}$ & 0.02 & 0.04 & 0.02 & 0.04 & 0.89 & 0.13 & 0.24 & $\mathrm{ND}$ & $\mathrm{ND}$ & 0.07 & 0.06 & 0.2 \\
\hline $\mathrm{MnO}$ & 0.03 & 0.03 & 0.02 & 0.03 & 0.13 & 0.06 & 0.05 & 0.02 & 0.02 & 0.07 & 0.08 & 0.09 \\
\hline $\mathrm{Cr}_{2} \mathrm{O}_{3}$ & 0.13 & $\mathrm{ND}$ & 0.15 & $\mathrm{ND}$ & $\mathrm{ND}$ & $\mathrm{ND}$ & $\mathrm{ND}$ & ND & $\mathrm{ND}$ & $\mathrm{ND}$ & $\mathrm{ND}$ & 0.06 \\
\hline $\mathrm{SO}_{3}$ & 0.02 & 0.04 & ND & $\mathrm{ND}$ & 0,10 & $\mathrm{ND}$ & 0,01 & 0,04 & 0,01 & 0,02 & 0,04 & 0.07 \\
\hline LOI & 48.14 & 40.03 & 42.07 & 38.52 & 4.20 & 1.43 & 1.44 & $\mathrm{ND}$ & 1.05 & 6.68 & 6.53 & 7.29 \\
\hline TOTAL & 99.96 & 99.91 & 99.97 & 99.96 & 99.81 & 99.85 & 99.84 & 99.97 & 100,00 & 99.93 & 99.96 & 99.83 \\
\hline Trace Element (ppm) & F41 & F41_A & F42_A & F42_B & F45 & F46_47 & F48 & F49 & F50 & F52 & $\mathbf{F 5 3}$ & F54 \\
\hline As & 15 & 115 & 96 & 155 & 256 & 466 & 727 & 14 & $\mathrm{ND}$ & 13 & ND & 103 \\
\hline $\mathrm{Ba}$ & ND & 417 & 320 & 418 & 706 & 293 & 494 & 622 & $\mathrm{ND}$ & 514 & 817 & 800 \\
\hline $\mathrm{Br}$ & ND & ND & ND & ND & ND & ND & $\mathrm{ND}$ & ND & ND & $\mathrm{ND}$ & $\mathrm{ND}$ & ND \\
\hline $\mathrm{Ce}$ & ND & $\mathrm{ND}$ & ND & ND & ND & 279 & 369 & $\mathrm{ND}$ & ND & ND & ND & ND \\
\hline $\mathrm{Cl}$ & 311 & ND & 160 & 194 & 96 & ND & 107 & ND & 114 & 154 & 300 & 132 \\
\hline Co & ND & ND & ND & ND & ND & ND & 15 & ND & 68 & 1 & $\mathrm{ND}$ & 8 \\
\hline $\mathrm{Cu}$ & 23 & 17 & 23 & $\mathrm{ND}$ & 91 & 25 & 40 & 29 & 32 & 22 & 28 & 37 \\
\hline $\mathrm{Ga}$ & $\mathrm{ND}$ & $\mathrm{ND}$ & ND & $\mathrm{ND}$ & $\mathrm{ND}$ & ND & 17 & 17 & $\mathrm{ND}$ & $\mathrm{ND}$ & ND & ND \\
\hline $\mathrm{Ge}$ & ND & $\mathrm{ND}$ & ND & $\mathrm{ND}$ & $\mathrm{ND}$ & ND & $\mathrm{ND}$ & $\mathrm{ND}$ & $\mathrm{ND}$ & $\mathrm{ND}$ & ND & ND \\
\hline Mo & ND & ND & 21 & ND & ND & ND & ND & $\mathrm{ND}$ & $\mathrm{ND}$ & ND & $\mathrm{ND}$ & ND \\
\hline $\mathrm{Nb}$ & $\mathrm{ND}$ & $\mathrm{ND}$ & $\mathrm{ND}$ & $\mathrm{ND}$ & $\mathrm{ND}$ & 18 & 13 & $\mathrm{ND}$ & ND & $\mathrm{ND}$ & $\mathrm{ND}$ & ND \\
\hline $\mathrm{Ni}$ & 15 & 14 & 17 & 16 & 26 & 21 & 78 & 18 & 25 & $\mathrm{ND}$ & 20 & 282 \\
\hline $\mathrm{Pb}$ & $\mathrm{ND}$ & $\mathrm{ND}$ & $\mathrm{ND}$ & $\mathrm{ND}$ & $\mathrm{ND}$ & 75 & 50 & $\mathrm{ND}$ & $\mathrm{ND}$ & $\mathrm{ND}$ & ND & ND \\
\hline $\mathrm{Rb}$ & ND & 6 & 9 & 18 & 151 & 395 & 301 & 74 & 0 & 98 & 129 & 123 \\
\hline $\mathrm{Se}$ & ND & $\mathrm{ND}$ & ND & ND & $\mathrm{ND}$ & $\mathrm{ND}$ & ND & $\mathrm{ND}$ & 37 & $\mathrm{ND}$ & $\mathrm{ND}$ & ND \\
\hline $\mathrm{Sr}$ & 1243 & 291 & 153 & 154 & 218 & 19 & 87 & 50 & 29 & 29 & 34 & 114 \\
\hline Th & 149 & $\mathrm{ND}$ & 20 & 21 & 21 & 29 & 53 & $\mathrm{ND}$ & $\mathrm{ND}$ & $\mathrm{ND}$ & $\mathrm{ND}$ & ND \\
\hline $\mathrm{U}$ & ND & ND & ND & ND & ND & ND & $\mathrm{ND}$ & ND & $\mathrm{ND}$ & $\mathrm{ND}$ & ND & ND \\
\hline V & ND & ND & ND & 33 & 458 & 27 & ND & $\mathrm{ND}$ & $\mathrm{ND}$ & $\mathrm{ND}$ & ND & $\mathrm{ND}$ \\
\hline $\mathrm{Y}$ & ND & $\mathrm{ND}$ & ND & $\mathrm{ND}$ & $\mathrm{ND}$ & $\mathrm{ND}$ & $\mathrm{ND}$ & $\mathrm{ND}$ & $\mathrm{ND}$ & $\mathrm{ND}$ & ND & ND \\
\hline $\mathrm{Yb}$ & ND & $\mathrm{ND}$ & $\mathrm{ND}$ & $\mathrm{ND}$ & ND & ND & $\mathrm{ND}$ & $\mathrm{ND}$ & ND & $\mathrm{ND}$ & $\mathrm{ND}$ & ND \\
\hline $\mathrm{Zn}$ & $\mathrm{ND}$ & 24 & 15 & 24 & 88 & 60 & 62 & 40 & 22 & 38 & 40 & ND \\
\hline $\mathrm{Zr}$ & ND & 193 & 14 & 34 & 79 & 523 & 286 & 101 & ND & 138 & 138 & 150 \\
\hline
\end{tabular}

\section{Fluid inclusion study}

Microthermometric measurements were carried out from two-phase primary inclusions (Figure 5), taking into account the criteria specified by Roedder (1984) and Van den Kerkhof and Hein (2001), among the magnesites in the fault zone, where U-Th mineralization was highest in the study area (Table 2). Liquid inclusions are mostly irregular and contain liquid and vapor $(\mathrm{L}-\mathrm{V})$ at room temperature (Figure 5). In the measurements at these inclusions, all homogenisations occurred in the liquid phase. Although the bubbles of the $\mathrm{V}$ phase are quite large in some inclusions (Figure 
5a), they are generally small in size (Figure 5b). Using the equation of Bodnar (1993), the melting temperatures obtained for each inclusion were calculated from the measurements, $\% \mathrm{NaCl}$ equivalents of the inclusion (Table 2). In the moderate of the data got from the liquid inclusions, the density of the inclusions was calculated according to Zhang and Frantz (1987) with the service of the BULK (Bakker, 2003) package program (Table 2). Because of the calculations, it was demonstrated that the density of the liquids ranged between $0.58-0.74 \mathrm{~g} / \mathrm{cm}^{3}$ (Table 2).

Table 2. Microthermometric data from magnesites.

\begin{tabular}{cccccccc}
\hline $\begin{array}{c}\text { Sample } \\
\text { No }\end{array}$ & Mineral & Type & $\begin{array}{c}\text { Eutectic } \\
\text { Temperature }\left(\mathbf{T e}^{\mathbf{0}} \mathbf{C}\right)\end{array}$ & $\begin{array}{c}\text { Final Ice } \\
\text { Melting } \\
\text { Temperatures } \\
\left(\text { Tm-ice }^{\mathbf{0}} \mathbf{C}\right)\end{array}$ & $\begin{array}{c}\text { Homogenization } \\
\text { Temperature } \\
\left(\mathbf{T h}^{\mathbf{0}} \mathbf{C}\right)\end{array}$ & $\begin{array}{c}\text { \% NaCl } \\
\text { Salinity }\end{array}$ & ${\text { Density }(\mathbf{d}) \mathbf{g} \mathbf{c m}^{\mathbf{3}}}$ \\
\hline 1 & Magnesite & LV & -47.2 & -3.8 & 298 & 6.2 & 0.70 \\
2 & Magnesite & LV & -46.3 & -4.8 & 348 & 7.6 & 0.58 \\
3 & Magnesite & LV & -44.6 & -2.5 & 315 & 4.2 & 0.66 \\
4 & Magnesite & LV & -46.8 & -4.6 & 341 & 7.3 & 0.60 \\
5 & Magnesite & LV & -38.6 & -5.1 & 283 & 8.0 & 0.74 \\
6 & Magnesite & LV & -29.5 & -2.9 & 315 & 4.8 & 0.66 \\
7 & Magnesite & LV & -34.3 & -3.1 & 334 & 5.1 & 0.61 \\
8 & Magnesite & LV & -35.5 & -4.9 & 342 & 7.7 & 0.59 \\
9 & Magnesite & LV & -40.3 & -3.9 & 322 & 6.3 & 0.65 \\
10 & Magnesite & LV & -46.2 & -4.1 & 320 & 6.6 & 0.65 \\
11 & Magnesite & LV & -46.8 & -2.6 & 289 & 4.3 & 0.72 \\
12 & Magnesite & LV & -44.2 & -4.1 & 345 & 6.6 & 0.58 \\
13 & Magnesite & LV & -43.5 & -3.9 & 282 & 6.3 & 0.74 \\
14 & Magnesite & LV & -39.1 & -3.1 & 312 & 5.1 & 0.67 \\
15 & Magnesite & LV & -45.5 & -3.9 & 329 & 6.3 & 0.63 \\
\hline
\end{tabular}
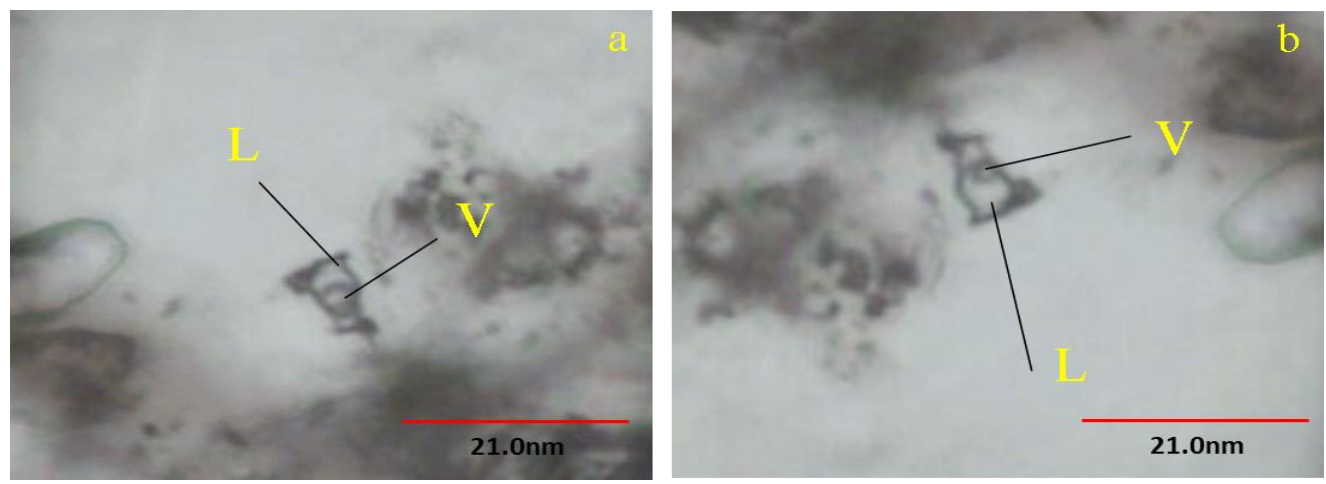

Figure 5. The photomicrographs of liquid-gas (LV Type) phases of primary inclusions observed in magnesites.

In measurements of LV-type inclusions in magnesite samples in the northwest of Arrkl1, where $\mathrm{U}$ mineralization is observed, Tm-ice values are between $-2.5{ }^{\circ} \mathrm{C}$ and $-5.1{ }^{\circ} \mathrm{C}$ and salinity equivalents $(\% \mathrm{NaCl})$ (Bodnar, 1993) are between 4.2-8.0. Homogenization temperatures $\left(\mathrm{Th},{ }^{\circ} \mathrm{C}\right)$ are between $282-348{ }^{\circ} \mathrm{C}$. It is seen that the eutectic temperature values $\left(\mathrm{Te},{ }^{\circ} \mathrm{C}\right)$ are between $-29.5{ }^{\circ} \mathrm{C}$ and $-47.2{ }^{\circ} \mathrm{C}$, while the final ice melting temperature values are relatively $\left(\mathrm{Tm}\right.$-ice, $\left.{ }^{\circ} \mathrm{C}\right)$ in narrower intervals (Figure 6). 


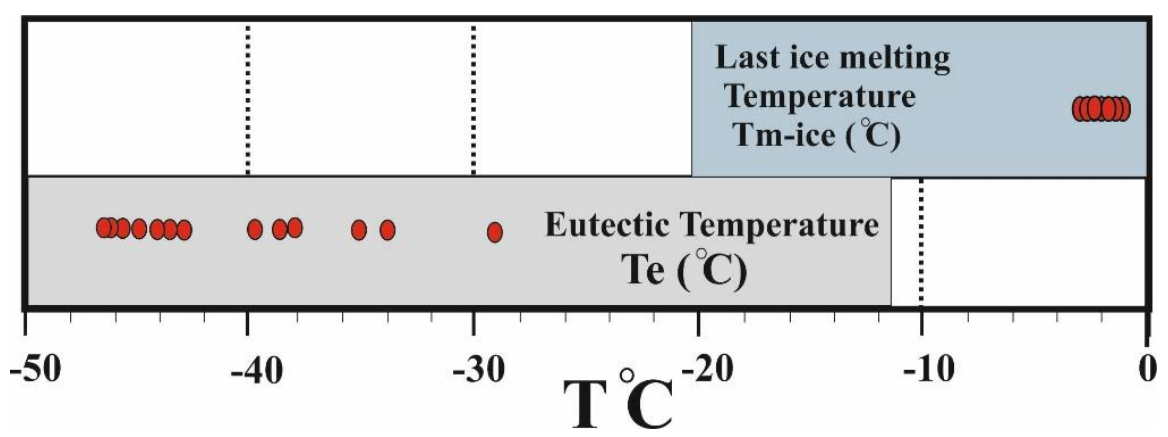

Figure 6. Distribution of microthermometric values detected in magnesites.

When the homogenization temperature $\left(\mathrm{Th},{ }^{\circ} \mathrm{C}\right)$ and salinity (\% $\mathrm{NaCl}$ equivalent) values are evaluated, the salinity values are between 4.2-8.0 (average: 6.2), and the homogenization temperatures $\left(\mathrm{Th},{ }^{\circ} \mathrm{C}\right)$ values are between $282-348$ ${ }^{\circ} \mathrm{C}$ (average: $318{ }^{\circ} \mathrm{C}$ ) as seen in frequency histogram (Figure 7). While homogenization temperatures are encountered in wide ranges in $\mathrm{U}$ Th mineralization, $\% \mathrm{NaCl}$ salinity equivalents exhibit a tighter distribution than homogenization temperatures (Figure 7).

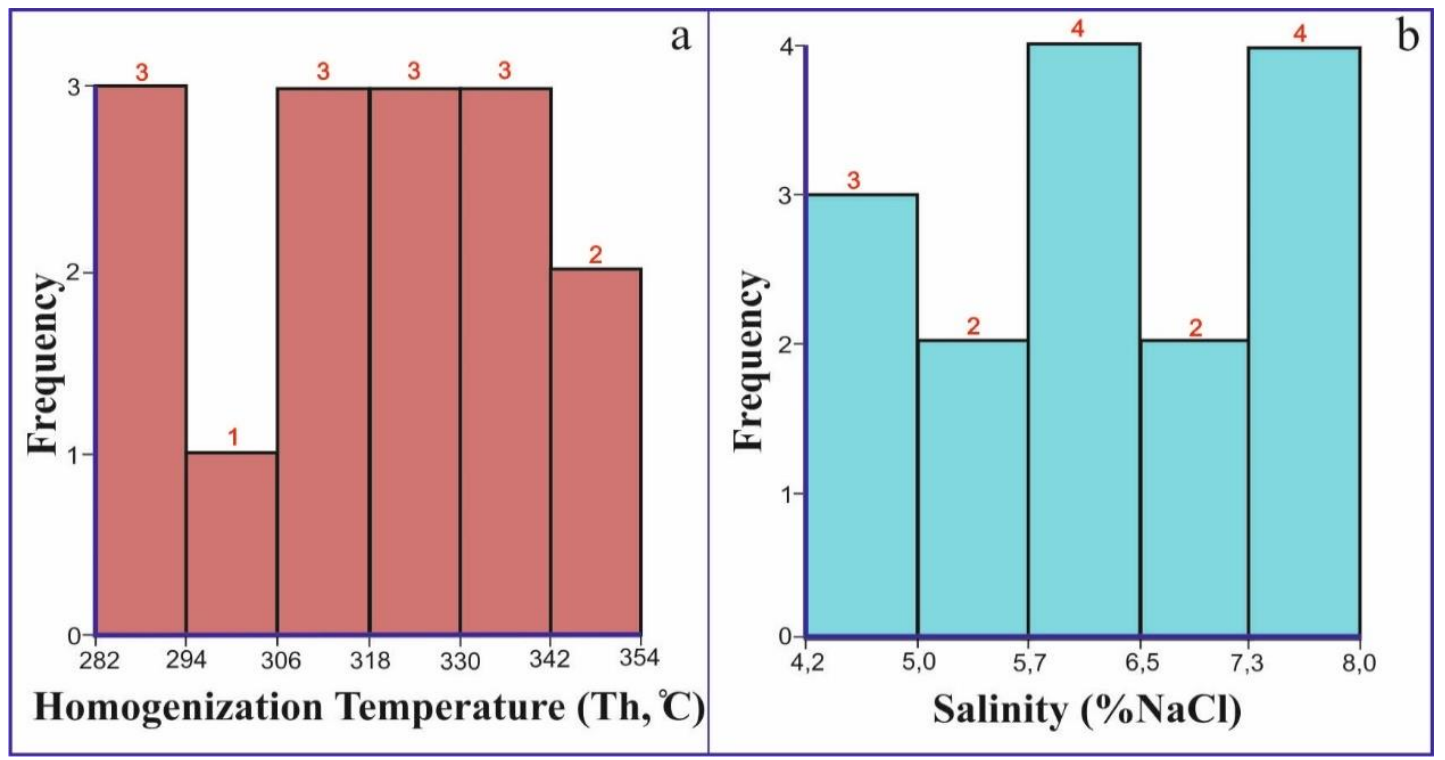

Figure 7. Frequency histograms of inclusions observed in magnesite; a. Homogenization Temperature (The, $\left.{ }^{\circ} \mathrm{C}\right)$, b. Salinity (\% $\mathrm{NaCl}$ Equivalent).

Microthermometric data obtained in ore zones were compared with data obtained in various deposits in the world (Figure 8). According to the data obtained, it was determined that the mineralization was homogenized at higher temperatures close to the epithermal phase, and the salinity values showed a similar distribution to this phase.

The microthermometric measurement values detected in hydrothermal magnesites with uranium and thorium mineralization were evaluated according to the tables developed by Roedder (1979) and Shephard et al. (1985). Accordingly, eutectic temperature values $\left(\mathrm{Te},{ }^{\circ} \mathrm{C}\right)$ show that the solution is rich in $\mathrm{CaCl}_{2}$ and $\mathrm{MgCl}_{2}$ (Crawford,
1981; Roedder, 1984). As a result of these data, the solution system is $\mathrm{H}_{2} \mathrm{O}-\mathrm{MgCl}_{2}-\mathrm{CaCl}_{2}$.

\section{Discussion and conclusion}

The important uranium mineralization is generally sedimentary type in Turkey (Akıska et al., 2019), only Demirtepe (Söke-Aydın) as vein-type mineralization (TÜSİAD, 1998). In Demirtepe, uranium enrichment is observed in fault zones developing in Paleozoic aged schists (Akiska et al., 2019). Thorium deposits, located in KizılcaörenEskişehir (Turkey), formed in barite, fluorite and REE deposit (Kaplan, 1977). In addition, thorium occurances were found in Malatya-DarendeKuluncak, Kayseri-Felahiye, Sivas and Diyarbakır regions (DPT, 1996; Akkoyunlu, 2006). 


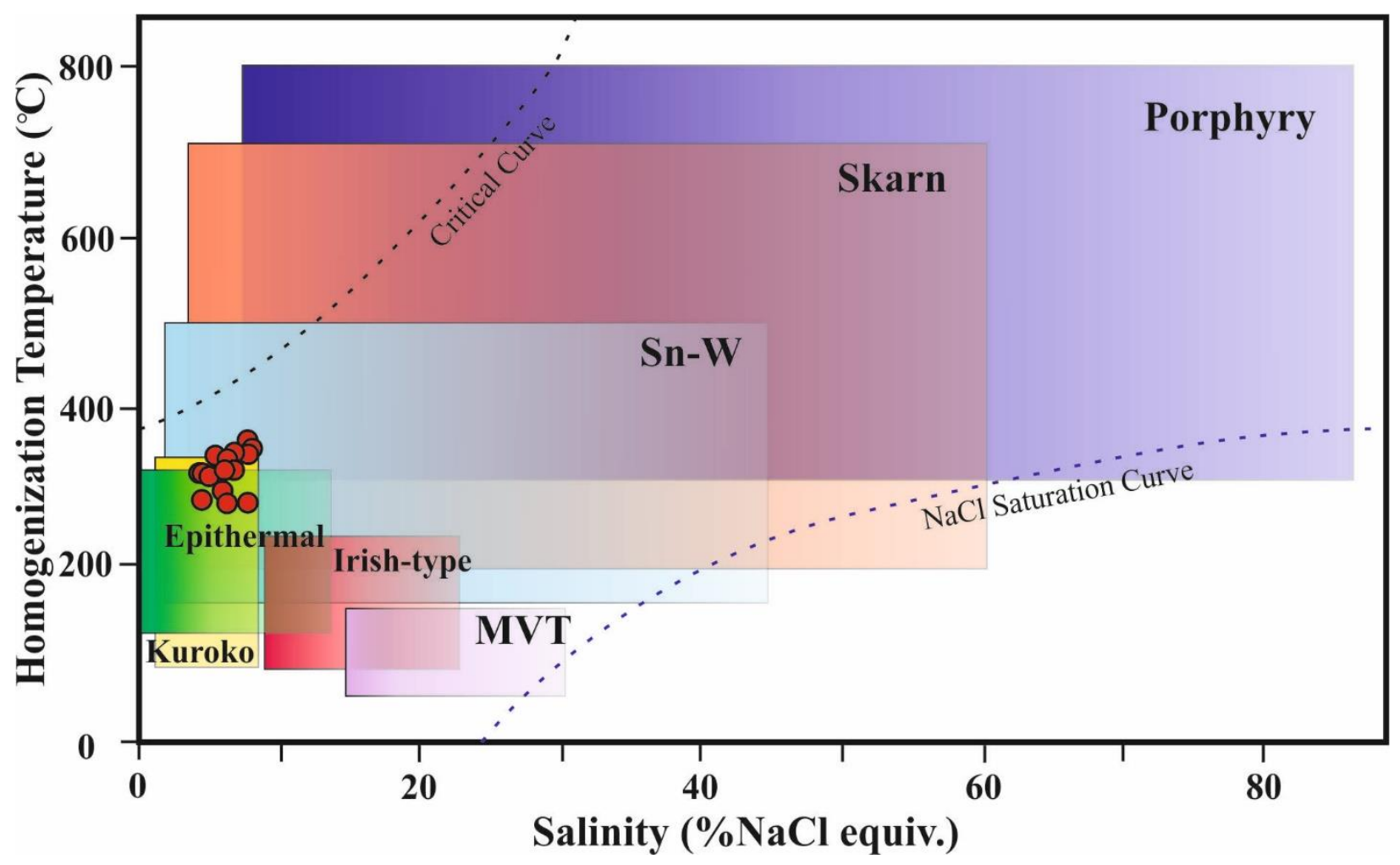

Figure 8. Comparison of homogenization temperature-salinity values detected in magnesites with various deposits in the world (Wilkinson, 2001).

With the effect of the stress system in the Paleogene period in the study area, dip-slip fault systems have developed. These fault zones are mostly E-W and NE-SW trending. U-Th mineralization observed in fault zones in volcanoclastic rocks in the northwest of Ariklı is epigenetic and vein type (Günaydın, 2017). The highest U-Th values are in the area with hydrothermal magnesites.

Fluid inclusion studies in ore zones that are thought to be in hydrothermal systems provide critical information (Lu et al., 2004a; Chen et al., 2007). It is possible to evaluate together with the geology and geochemistry of the region. Li et al. (2002) stated that many uranium deposits in Southern China are related with faults and that faults perform significant appearances in the formation and distribution of hydrothermal uranium deposits.

In an extensional tectonic environment, revealing the physicochemical properties of ore-forming fluids is an important component to inspect the genesis of mineralization. Presumably, the fluids existing to the bed rocks rich in $\mathrm{U}$ and $\mathrm{Th}$ were mobilized and deposited in convenient areas depending on the fault activity. A complementary situation is also observed in U ores in Southern China (Bonnetti et al., 2018; Shu et al., 2009; Wang and Shu, 2012). The fluids belonging to the bedrock in the $\mathrm{U}$ fields established by the stress tectonic system in Southern China were mobilized after faulting and also deposited in sufficient physicochemical environments (Riegler et al., 2014; Bonnetti et al., 2020).

In this study, hydrothermal magnesites in the same fracture line as the ore zone exhibit high homogenization temperatures $\left(282-348{ }^{\circ} \mathrm{C}\right.$, average: $\left.318{ }^{\circ} \mathrm{C}\right)$ and low salinity (4.2-8.0\% weight $\mathrm{NaCl}$ equivalent). Because of the results obtained from the inclusions, it was decided that the density of the liquids were between $0.58-0.74 \mathrm{~g} / \mathrm{cm}^{3}$.

The geology, geochemsitry and fluid inclusion characteristics of the mineralisation in the Arıklı UTh deposit are consistent with a hydrothermal origin. Microthermometric data obtained in ore zones show that mineralization was homogenized at higher temperatures close to the epithermal phase and salinity values showed a similar distribution to this phase.

\section{References}

Akıska, E., Karakaş, Z. and Öztürk, C. (2019). Uranium, thorium and rare earth element deposits of Turkey. Pirajno, F., Ünlü, T., Dönmez, C., Şahin, M. (Eds.). Mineral Resources of Turkey (p. 655679). Crawley; Ankara: Springer, Cham., 16. https://doi.org/10.1007/978-3-030-02950-0_14. 
Akkoyunlu, A. (2006). Türkiye'de enerji kaynakları ve çevreye etkileri [Energy sources and their environmental effects in Turkey]. Turkish Asian Center for Strategic Studies.

Andaç, M. (1971). Biga yarımadasında tarihi Truva harabelerinin güneyindeki radyoaktif sahil kumlarının mineralojisi ve bunların ana kayaçlarının petrolojisi. MTA Dergisi, 95, 75-79.

Anonymous. (2009). World distribution of uranium deposit (UDEPO) with uranium deposit classification. IAEA, TECDOC, 1629.

Atabey, E. (2006). Çanakkale'nin Ayvacık-Küçükkuyu arası kayaların özellikleri, MTA'nın uranyumfosfat aramaları ve doğal radyoaktivite. Radyasyon ve Çevre Sempozyumu Bildiriler kitabı (s.1), Çanakkale.

Atabey, E. ve Ünal, H. (2008). Batı Anadolu'daki jeolojik unsurlar ve halk să̆lı̆̆l projesi 20062007 yıl tıbbi jeoloji ve etüt raporu. Maden Tetkik ve Arama Genel Müdürlüğü, MTA, Rapor No, 11067, 292.

Atabey, E. (2008). Doğal radyasyon kaynakları: Çanakkale ili Ayvacık ve Geyikli örneği. Uluslararası Katılımlı Tıbbi Jeoloji Sempozyum Kitabı (s. 85-88). YMGV Yayın1. ISBN: 978975-7946-33-5, İstanbul.

Bakker, R.J. (2003). Package FLUIDS 1. Computer programs for analysis of fluid inclusion data and for modelling bulk fluid properties, Chemical Geology, 194, 3-23. https://doi.org/10.1016/S0009-2541(02)002681.

Beccaletto, L. and Jenny, C., (2004). Geology and correlation of the Ezine Zone: a Rhodope fragment in NW Turkey. Turkish Journal of Earth Science, 13(2), 145-176.

Beccaletto, L., Bartolini A.C., Martini R., Hochuli P.A. and Kozur H. (2005). Biostratigraphic data from the Çetmi melange, northwest Turkey. Palaeogeographic and tectonic implications, Palaeogeography, Palaeoclimatology, Palaeoecology, 221, 215-244. https://doi.org/10.1016/j.palaeo.2005.02.011.

Bodnar, R.J. (1993). Revised equation and table for determining the freezing point depression of $\mathrm{H}_{2} \mathrm{O}-\mathrm{NaCl}$ solutions. Geochemistry Cosmochemical Acta, 57, 683-684. https://doi.org/10.1016/0016-7037(93)90378-A.

Bonnetti, C., Liu, X., Mercadier, J., Cuney, M., Deloule, E., Villeneuve, J. and Liu, W. (2018). The genesis of granite-related hydrothermal uranium deposits in the Xiazhuang and Zhuguang ore fields, north Guangdong Province, SE China: Insights from mineralogical, trace elements and
$\mathrm{U}-\mathrm{Pb}$ isotopes signatures of the $\mathrm{U}$ mineralisation. Ore Geology Review, 92, 588-612. https://doi.org/10.1016/j.oregeorev.2017.12.010.

Bonnetti, C., Liu, X., Cuney, M., Mercadier, J., Riegler, T. and Yu. C. (2020). Evolution of the uranium mineralisation in the Zoujiashan deposit, Xiangshan ore field: Implications for the genesis of volcanic-related hydrothermal $\mathrm{U}$ deposits in South China. Ore Geology Reviews, 122, 103514, 1-24. https://doi.org/10.1016/j.oregeorev.2020.103514

Chen, Y.J., Ni, P., Fan, H.R. and Lai, Y. (2007). Diagnostic fluid inclusions of different types gold deposits. Acta Petrolei Sinica, 23, 20852108 (in Chinese with English abstract). http://cpfd.cnki.com.cn/Article/CPFDTOTALGDKL200711002057.htm.

Contencin, G. (1960). Sivrihisar Beylikahır radyoaktif emaresi [Radioactive signs of Sivrihisar Beylikahır]. General Directorate of Mineral Research and Exploration Report, no 3167 (in Turkish, unpublished).

Crawford, M. L. (1981). Phase equilibria in aqueous fluid inclusions. In Mineralogical Association of Canada Short Course Handbook. Hollister, L.S., Crawford, M.L. (Eds.), 6, 75-100.

Çiftçi, N.B., Temel, R.Ö. and Terzioğlu, M.N. (2004). Neogene stratigraphy and hydrocarbon system of the region surrounding the gulf of Edremit, NW Turkey. Turkish Association of Petroleum Geologists Bulletin, 16, 81-104 (in Turkish with English abstract).

DPT. (1996). Nükleer enerji hammaddeleri-UranyumToryum [Nuclear energy raw materialsuranium-thorium]. Turkish republic prime ministry state planning organization undersecretariat, Mining specialization commission-sub-commission on energy raw materials, Geothermal energy working group report, DPT 2429 (in Turkish, unpublished).

Dönmez, M., Akçay, A.E., Genç, S.C. and Acar, S. (2005). Middle-Upper Eocene volcanism and marine ignimbrites in Biga Peninsula. Bulletin Mineral Research Exploration, 131, 49-61 (in Turkish).

Günaydın, A. (2017). Geology and geochemistry of nodular-phosphate and fault-controlled hydrothermal-phosphate mineralizations in Arıklı and Nusratlı villages (Ayvacık-Çanakkale, NW Turkey). Bulletin of the Mineral Research and Exploration, 155 (155), 131-146. https://doi.org/10.19111/bulletinofmre.314197. 
Işık, V. (2016). Torosların Jeolojisi; Türkiye Jeolojisi Ders Notu. Ankara Üniversitesi, Jeoloji Mühendisliği Bölümü, Ankara.

İnci, U. (1984). The stratigraphy and organic properties of Demirci and Burhaniye bituminous shales. Bulletin of the Geological Congress of Turkey, 5 , 27-40 (in Turkish with English abstract).

Kaplan, H. (1977). Eskisehir-Sivrihisar-Kızılcaören köyü yakın güneyi nadir toprak elementleri ve toryum kompleks cevher yatağı nihai etüdü [Final report of the rare earth elements and thorium ore complex in Eskişehir-SivrihisarKizilcaören village]. General Directorate of Mineral Research and Exploration Report, no 8672 (in Turkish, unpublished).

Kaplan, H. (2011). Kızılcaören toryum/nte yatağının keşfi üzerine, kişisel söyleşi [On the discovery of the Kizılcaören Thorium/REE beds, personal interview]. Ministry of Earth Science Magazine, $13,52-62$.

Kesgin, Y. (2001). Tertiary geology and sedimentology of the northeastern Aegean offshore and nearshore regions. PhD Thesis, p.265, Ankara University, Ankara, Turkey (in Turkish with English abstract).

Li, J., Zhou, M., Li, X., Fu, Z. and Li, Z. (2002). Structural control on uranium mineralization in South China: Implications for fluid flow in continental strike-slip faults. Science in China (Series-D), 45, 851-864. https://doi.org/10.1007/BF02879519.

Lu, H.Z., Fan, H.R., Ni, P., Ou, G.X., Shen, K. and Zhang, W.H. (2004). Fluid Inclusions. SciencePress, Beijing (pp, 487, in Chinese).

MTA. (2009). Mineral resources of Turkey (According to Province). Publication of Mineral Research and Exploration, Earth Science and Culture Series-5, Ankara, p 602. ISBN 978-605-407532-4.

MTA. (2010c). Maden Tetkik ve Arama Genel Müdürlüğü websitesi, (Accessed 22 Oct 2015). http://www.mta.gov.tr/v2.0/turkiye_maden/ maden_potansiyel_2010/Canakkale_Madenler.p df.

Okay, A.I., Siyako, M. and Bürkan, K.A. (1990). Geology and tectonic evolution of the Biga peninsula. Association Of Turkish Petroleum Gelologists Bulletin, 2, 83-121.

Okay A.İ., and Satır M. (2000a). Upper Cretaceous Eclogite-Facies Metamorphic Rocks From the Biga Peninsula, Northwest Turkey. Turkish Journal of Earth Sciences, 9, 47-56.
Okay, A.İ. and Satır, M. (2000b). Coeval plutonism and metamorphism in a latest Oligocene metamorphic core complex in northwest Turkey. Geological Magazine, 137 (5), 495-516.

Okay, A. I., Tansel, I. and Tuysuz, O. (2001). Obduction, subduction and collision as reflected in the Upper Cretaceous-Lower Eocene sedimentary record of western Turkey. Geological Magazine, 138(2), 117-142.

Okay, A.I. and Altıner, D. (2004). Uppermost Triassic limestone in the Karakaya Complex stratigraphic and tectonic significance. Turkish Journal of Earth Sciences, 13,187-199.

Örgün, Y., Altınsoy, N., Gültekin, A. H., Karahan, G. and Çelebi, N. (2005). Natural radioactivity levels in granitic plutons and groundwaters in Southeast part of Eskisehir, Turkey. Applied Radiation and Isotopes, 63(2), 267-275. https://doi.org/https://doi.org/10.1016/j.apradiso .2005.03.008.

Qin, M. T. and Liu, S. X. (1998). Granite- and volcanic rocks-type uranium deposits in the Nanling region (in Chinese with English abstract), Beijing: Geological Publishing House, 147-155.

Riegler, T., Lescuyer, J.-L., Wollenberg, P., Quirt, D. and Beaufort, D. (2014). Alteration related to uranium deposits in the Kiggavik-Andrew lake structural trend, Nunavut, Canada: new insights from petrography and clay mineralogy. Canadian Mineralogy, 52, 27-45. https://doi.org/10.3749/canmin.52.1.27.

Roedder, E. (1979). Origin and significance of magmatic inclusions. Bulletin de Mineralogie, 102(5), 487-510. https://doi.org/10.3406/bulmi.1979.7299.

Roedder, E. (1984). Fluid inclusions. Reviews in mineralogy: Washington. Mineral Society of America, 12, 644 p.

Saka K. (1979). Edremit körfezi ve civarı Neojen'inin jeolojisi ve hidrokarbon olanaklar1. Türkiye Petrolleri Anonim Ortaklı̆̆ Arama Grubu, 1342, $1-17$.

Shephard, T.J., Rankin, A.H. and Alderton, D.H.M. (1985). A practical guide to fluid inclusion studies. Blackie, Glasgow, p.239.

Shu, L.S., Zhou, X.M., Deng, P., Wang, B., Jiang, S.Y., $\mathrm{Yu}$, J.H. and Zhao, X.X. (2009). Mesozoic tectonic evolution of the Southeast China Block: new insights from basin analysis. Journal of Asian Earth Sciences, 34, 376-391. https://doi.org/10.1016/j.jseaes.2008.06.004.

Siyako, M., Bürkan, K.A. ve Okay, A.İ. (1989). Biga ve Gelibolu Yarımadaları'nın Tersiyer jeolojisi ve 
hidrokarbon olanakları. Türkiye Petrol Jeologları Derneği Bülteni, 1, 183-200.

Şaşmaz, A. (2008). Determination of uranium occurrences from soil, water and plant samples and possible environmental effects around Köprübaşı (Manisa) uranium bed. Scientific and Technical Research Council of Turkey, Project no $107 \mathrm{Y} 226,82 \mathrm{p}$ (in Turkish, unpublished).

Şengün, F., Yiğitbaş, E. and Tunç, İ.O. (2011). Geology and tectonic emplacement of eclogite and blueschists, Biga peninsula, northwest Turkey. Turkish Journal of Earth Science, 20, 273-285. https://doi: 10.3906/yer-0912-75.

TÜSİAD. (1998). Evaluation of Turkey's energy strategy while entering the 21st century. Paper No. Tüsiad T/98-12/239, $316 \mathrm{p}$ (in Turkish, unpublished).

Uçmak, F. (1969). Final report of thorium ores in Eskişehir-Sivrihisar-Beylikahır. General Directorate of Mineral Research and Exploration Report, no 343 (in Turkish, unpublished).

Van den Kerkhof, A. M. and Hein, U.F. (2001). Fluid inclusion petrography. Lithos, 55(1-4), 27-47.

Wang, D. and Shu, L., (2012). Late Mesozoic basin and range tectonics and related magmatism in Southeastern China. Geoscience Frontiers, 3-2, 109-124.

https://doi.org/10.1016/j.gsf.2011.11.007.
Wilkinson, J. J. (2001). Fluid inclusions in hydrothermal ore deposits. Lithos, 55(1-4), 229-272. https://doi.org/10.1016/S0024-4937(00)000475.

Y1lmaz-Şahin, S., Güngör, Y. and Göker, A.F., (2004). Macroscopical and microscopical evidences magma mixing/mingling type interaction of in Kestenbol granitoid (South Çanakkale), Nortwest AnatoliaTurkey, 4. Internatilnal Scientific Conference, Modern Managment of Mine Producing, Geology and Environmental protection, SGEM 2004, Bulgaria, Proceeding, 314.

Yılmaz-Şahin, S., Örgün, Y. ve Güngör, Y. (2006). U/TH'ca zengin radyoaktif granitoyidlerin jeokimyası: Kestanbol ve Kaymaz plütonları, batı Anadolu, Türkiye. 59. Türkiye Jeoloji Kurultayı Bildiri Özleri, 43-45, Ankara.

Yörükoğlu, K. (2014). Uranium and uranium potential of Turkey as a raw material. Architect and Engineer Journal, 75, 40-42 (in Turkish).

Zhang, Y.G. and Frantz, J.D. (1987). Determination of the homogenization temperatures and densities of supercritical fluids in the system $\mathrm{NaCl}-\mathrm{KCl}$ $\mathrm{CaCl}_{2}-\mathrm{H}_{2} \mathrm{O}$ using synthetic fluid inclusions, Chemical Geology, 64, 335-350. https://doi.org/10.1016/0009-2541(87)90012-X.

Zhang, Z. H. and Zhang, B. T., (1991). Studies of Ubearing granites and associated uranium ore deposits in south China (in Chinese with English abstract), Beijing: Atomic Energy Publishing House, 70-105. 